\title{
Assessment of Growth in Pediatric Syrian Refugee Populations in Jordan
}

\author{
Amanda Vakos ${ }^{1,2, *}$ Nadia Khalil ${ }^{3, *(} \quad$ Ambuj Kumar $^{4}$ Lynette Menezes ${ }^{4}$ Minal Ahson ${ }^{5}$
}

${ }^{1}$ Morsani College of Medicine, University of South Florida, Tampa, Florida, United States

2 Department of Obstetrics and Gynecology, West Penn Hospital/Allegheny Health Network, Pittsburgh, Pennsylvania, United States

${ }^{3}$ Department of Neurology, Morsani College of Medicine, University of South Florida, Tampa, Florida, United States

${ }^{4}$ Department of Internal Medicine, Morsani College of Medicine, University of South Florida, Tampa, Florida, United States

${ }^{5}$ Department of Internal Medicine, Department of Pediatrics Morsani College of Medicine, University of South Florida, Tampa, Florida, United States

Avicenna J Med 2021;11:167-171.
Address for correspondence Amanda Vakos, MD, 4800 Friendship Ave, Pittsburgh, PA 15224, United States

(e-mail: amanda.vakos@ahn.org).

\begin{abstract}
Keywords

- global health

- pediatrics

- growth

- nutrition

- syrian refugee crisis

- syrian refugees

Background Externally displaced pediatric Syrian refugees are at risk of adverse developmental health outcomes in the setting of food insecurity. To evaluate the impact of displacement on nutritional status of Syrian children, this study describes the prevalence of malnutrition of Syrian children residing in Jordan.

Methods A retrospective cross-sectional analysis of data from patient records $<18$ years of age from the Syrian American Medical Society's (SAMS) January 2019 mission trip to Jordan was performed. Prevalence of stunting, severe wasting, wasting, overweight, and underweight were calculated using the World Health Organization (WHO) definitions.

Results Overall, 392 Syrian children were included. In the 0 to 59 months group $(n=191)$, prevalence of stunting was $13.9 \%(n=165)$, severe wasting $4.2 \%(n=165)$, wasting $6 \%(n=165)$, overweight $16.4 \%(n=165)$, and underweight $5.3 \%(n=190)$. In the $>59$ months group ( $n=201)$, prevalence of stunting was $7.2 \%(n=194)$, severe wasting $3.6 \%(n=194)$, wasting $5.2 \%(n=194)$, overweight $6.7 \%(n=194)$, and underweight $4.6 \%(n=153)$. Age group was significantly associated with stunting (odds ratio $[\mathrm{OR}]=2.08,95 \% \mathrm{Cl} 1.03$ to 4.19 ) and overweight $(\mathrm{OR}=2.72 ; 95 \% \mathrm{Cl} 1.36$ to 5.47) with increased odds of each in the 0-59 months group.

Conclusion The findings suggest evidence of higher prevalence of malnutrition in Syrian children compared with prior assessments and greater impact on the younger cohort. In 0 to 59 months' old Syrian children, stunting and wasting were of medium severity and overweight was of high severity. This may reflect food insecurity in the setting of ongoing conflict, strain on resources, and evolving refugee policies, acknowledging study limitations. Syrian refugee children living in Jordan experience wasting and stunting. Comprehensive assessment of current nutritional status of Syrian children is needed. Access to adequate and nutritious food is paramount.
\end{abstract}

These authors contributed equally to the manuscript (co-first authors).

published online

November 15, 2021
DOI https://doi.org/ $10.1055 / \mathrm{s}-0041-1736544$. ISSN 2231-0770.
(C) 2021. Syrian American Medical Society. All rights reserved. This is an open access article published by Thieme under the terms of the Creative Commons Attribution-NonDerivative-NonCommercial-License, permitting copying and reproduction so long as the original work is given appropriate credit. Contents may not be used for commercial purposes, or adapted, remixed, transformed or built upon. (https://creativecommons.org/ licenses/by-nc-nd/4.0/)

Thieme Medical and Scientific Publishers Pvt. Ltd., A-12, 2nd Floor, Sector 2, Noida-201301 UP, India 


\section{Introduction}

The Syrian refugee crisis encompasses the political, social, and economic turmoil and concomitant violence, injustice, and instability since the outbreak of civil war in $2011 .^{1}$ As of March 2019, there were 5,689,127 externally displaced Syrian refugees, $45 \%$ of which are children. ${ }^{2}$ Despite governmental and international efforts, refugees face limited access to basic resources, including food insecurity and reduced food diversity, posing substantial risk for adverse developmental health outcomes in pediatric populations. ${ }^{3-8}$

To evaluate the impact of displacement on nutritional status of Syrian children, this study describes the prevalence of acute malnutrition, stunting, and overweight among Syria children residing in Jordan.

\section{Materials and Methods}

The data used in this analysis were extracted and provided by members of the Syrian American Medical Society (SAMS) through retrospective review of patient charts from the January 2019 mission trip to Jordan. The study was approved by University of South Florida's Institutional Review Board, protocol number 00039351.

\section{Inclusion/Exclusion}

Syrian children $<18$ years of age between January 19 and 24 , 2019 , living in Jordan and seen by a pediatrician at one of six locations, including Mafraq, Maa'daba, Irbid, Saltt, Nuzha, and Marka, were eligible for inclusion. Children seen by general practitioners were excluded due to lack of logistics to collect anthropometric data. Additionally, children may have been included more than once, as data were collected over a 6-day period in which there were no measures in place to account for repeat visits.

\section{Data Abstraction}

Data was abstracted from all eligible subjects and included age in months, sex, weight, and height. Age was determined by birthdate on United Nations High Commissioner for Refugees (UNHCR) registration card; if unavailable, birthdate was provided by parent or another accompanying guardian, and if exact birthdate was unknown, guardians provided estimated age. Weight was measured by Smart Weight DVS digital scale without removal of clothes given cold weather. Length was measured by tape measure in the standing or recumbent position, which can result in a difference of approximately $0.7 \mathrm{~cm} .{ }^{9}$ All data were collected and recorded by SAMS volunteer physicians and nurse practitioners.

For patients between 0 to 59 months of age, BMI and $z$ scores were generated using the World Health Organization (WHO) Analyser. ${ }^{10}$ For patients $>59$ months of age, body mass index (BMI) and $z$ scores were generated using the WHO AnthroPlus calculator. ${ }^{11}$

Measurements for severe wasting, wasting, underweight, overweight, and stunting were calculated according to the WHO definitions. Severe wasting is defined as weight-forheight $<$ three standard deviations (SD) below the norm.
Wasting is defined as weight-for-height $<$ two SD below the norm. Underweight defined as weight-for-age $<$ two SD below the norm. Overweight is defined as weight-for-height $>$ two SD above the norm. Stunting is defined as height-forage $<$ two SD below the norm. ${ }^{12}$

We compared the prevalence of wasting, stunting, and overweight in our study to the WHO prevalence thresholds stipulated in a study published by Onis et al to determine severity of malnutrition in Syrian refugee children $<18$ years seen at SAMS clinics in Jordan. ${ }^{13}$

Descriptive statistics were used to summarize subject characteristics where continuous variables were summarized as medians with ranges and categorical variables as rates. The association between the outcome variable (e.g., stunting, overweight, etc.) and the predictor variable (e.g., age group) was assessed using binary logistic regression and summarized as odds ratio (OR) along with 95\% confidence intervals (CI). The statistical significance was set at $5 \%$ for all comparisons. All data analyses were performed using SPSS version 25 statistical analysis software. ${ }^{14}$

\section{Results}

Overall, 392 subjects met inclusion criteria. Of the 392 subjects included in this study, 191 (49\%) were 0 to 59 months of age and 201 (51\%) were > 59 months of age.

In 0 to 59-months age group, the median age was 24 months (range 0.79-59 months); 48\% $(n=92)$ were male, $51 \%(n=98)$ were female, and $1 \%(n=1)$ data for sex was missing. Prevalence of severe wasting, wasting, underweight, overweight, and stunting is reported in -Table 1. Notably, length was missing for 26 participants.

In the $>59$ months age group, the median age was 96 months (range 60-213 months); 54\% were male $(n=108)$ and $46 \%(n=93)$ were female. Prevalence of severe wasting, wasting, underweight, overweight, and stunting is reported in -Table 1. Notably, length was missing for 7 subjects of this group. Additionally, underweight was unable to be calculated for those more than 120 months of age, which were 42 subjects out of the $201>59$ months.

As shown in - Table 2, overall, age group was significantly associated with stunting (OR 2.08; 95\% CI 1.03 to 4.19; $p=0.04$ ) and overweight (OR 2.72; 95\% CI 1.36 to 5.47; $p=0.005$ ), where OR refers to ratio of the odds of the event in the 0 to 59 months age group to the odds of the event in the $>59$ months age group. Age group was not significantly associated with severe wasting (OR 1.18 ; $95 \% \mathrm{CI} 0.41$ to 3.45; $p=0.757$ ), wasting (OR $1.18 ; 95 \% \mathrm{CI} 0.48$ to $2.93 p=0.709$ ), or underweight (OR $1.1595 \% \mathrm{CI} 0.43$ to $3.12 ; p=0.77$ ).

\section{Discussion}

The WHO defines prevalence thresholds for stunting, wasting, and overweight for children $<5$ years for the purpose of global monitoring of malnutrition. The aim is for all countries to achieve "low" or "very-low" levels, and for governments and aid organizations to trigger action and target programs when prevalence thresholds are above these levels. This 
Table 1 Prevalence of severe wasting ${ }^{a}$, wasting ${ }^{b}$, underweight ${ }^{c}$, overweight ${ }^{d}$, and stunting ${ }^{e}$, in the 0 to 59 months and $>59$ months age groups

\begin{tabular}{|c|c|c|c|c|}
\hline \multirow[t]{2}{*}{ Variable } & \multirow[t]{2}{*}{ Event/total $^{\mathrm{f}}$} & \multirow[t]{2}{*}{ Proportion } & \multicolumn{2}{|c|}{ Simple $z$ 95\% confidence intervals } \\
\hline & & & Lower limit & Upper limit \\
\hline \multicolumn{5}{|l|}{ Overall } \\
\hline Stunting & $37 / 359$ & $10.30 \%$ & $7.16 \%$ & $13.45 \%$ \\
\hline Wasting & $20 / 359$ & $5.57 \%$ & $3.19 \%$ & $7.94 \%$ \\
\hline Severe wasting & $14 / 359$ & $3.89 \%$ & $1.89 \%$ & $5.90 \%$ \\
\hline Overweight & $40 / 359$ & $11.14 \%$ & $7.88 \%$ & $14.39 \%$ \\
\hline Underweight ${ }^{g}$ & $17 / 343$ & $4.95 \%$ & $2.65 \%$ & $7.25 \%$ \\
\hline \multicolumn{5}{|l|}{ 0-59 months } \\
\hline Stunting & $23 / 165$ & $13.93 \%$ & $8.65 \%$ & $19.22 \%$ \\
\hline Wasting & $10 / 165$ & $6.06 \%$ & $2.41 \%$ & $9.70 \%$ \\
\hline Severe wasting & $7 / 165$ & $4.24 \%$ & $1.16 \%$ & $7.31 \%$ \\
\hline Overweight & $27 / 165$ & $16.36 \%$ & $10.71 \%$ & $22.00 \%$ \\
\hline Underweight & $10 / 190$ & $5.26 \%$ & $2.08 \%$ & $8.43 \%$ \\
\hline \multicolumn{5}{|l|}{$>59$ months } \\
\hline Stunting & $14 / 194$ & $7.21 \%$ & $3.57 \%$ & $10.85 \%$ \\
\hline Wasting & $10 / 194$ & $5.15 \%$ & $2.04 \%$ & $8.26 \%$ \\
\hline Severe wasting & $7 / 194$ & $3.60 \%$ & $0.98 \%$ & $6.23 \%$ \\
\hline Overweight & $13 / 194$ & $6.70 \%$ & $3.18 \%$ & $10.21 \%$ \\
\hline Underweight ${ }^{g}$ & $7 / 153$ & $4.57 \%$ & $1.26 \%$ & $7.88 \%$ \\
\hline
\end{tabular}

Abbreviations: BMI, body mass index; SD, standard deviation.

a Severe wasting is defined as a $z$ score-for-BMI of $<-3$ SD.

bWasting is defined as a $z$ score-for-BMI of $<-2$ SD.

'Underweight is defined as a $z$ score-for-weight of $<-2$ SD.

${ }^{\mathrm{d}}$ Overweight is defined as a $z$ score-for-BMI of $>+2$ SD.

eStunting is defined as a $z$ score-for-length of $<-2$ SD.

'The differences in denominators are varied due to missing data as specified in the "Results" section. Each parameter is defined by specific anthropometric input, as per the WHO definitions, and calculator, as stipulated in the "Methods" section. Thus, some parameters are mutually exclusive while others are not.

${ }^{9}$ The differences in denominators for underweight in $>59$ months are because underweight cannot be calculated for children $>120$ months as described in the "Results" section.

Table 2 Association between age group (0-59vs. $>59$ months) and severe wasting ${ }^{\mathrm{a}}$, wasting ${ }^{\mathrm{b}}$, underweight ${ }^{\mathrm{c}}$, overweight ${ }^{\mathrm{d}}$, and stunting ${ }^{\mathrm{e}}$

\begin{tabular}{|l|l|l|}
\hline \multirow{2}{*}{} & \multicolumn{2}{|l|}{$\begin{array}{l}\text { Age group (0-59 vs. }>59 \\
\text { months) }\end{array}$} \\
\cline { 2 - 3 } & OR $^{\text {f } ; 95 \% ~ C l ~}$ & p-Value \\
\hline Severe wasting & $1.18 ;(0.41-3.45)$ & 0.757 \\
\hline Wasting & $1.18 ;(0.48-2.93)$ & 0.709 \\
\hline Underweight & $1.15 ;(0.43-3.12)$ & 0.77 \\
\hline Overweight & $2.72 ;(1.36-5.47)$ & 0.005 \\
\hline Stunting & $2.08 ;(1.03-4.19)$ & 0.04 \\
\hline
\end{tabular}

Abbreviations: $\mathrm{BMI}$, body mass index; $\mathrm{Cl}$, confidence interval; OR, odds ratio; SD, standard deviation.

${ }^{a}$ Severe wasting is defined as a $z$ score-for-BMI of $<-3$ SD.

bWasting is defined as a $z$ score-for-BMI of $<-2$ SD.

'Underweight is defined as a z score-for-weight of $<-2$ SD.

${ }^{\mathrm{d}}$ Overweight is defined as a $\mathrm{z}$ score-for-BMI of $>+2$ SD.

eStunting is defined as a $z$ score-for-length of $<-2$ SD.

fOR refers to ratio of the odds of the event in the 0 to 59 months age group to the odds of the event in the $>59$ months age group. study found the overall prevalence of stunting $(10.3 \%[95 \% \mathrm{CI}$, $7.16 \%, 13.45 \%]$ ) among Syrian refugee children age 0 to 59 months to be of medium severity, as defined by WHO prevalence thresholds. This is higher than the prevalence of stunting reported by a nutritional profile conducted by the International Organization for Migration (IOM) from 20152016, which found the prevalence of stunting in Syrian refugee children aged between 6 to 59 months to be 7.5\% (95\% CI, 6.9\%, 8.2\%), similar to another nutritional profile conducted from 2013-2014 in Jordan, Lebanon, and Iraq, which reported a prevalence of $10.5 \%$ (95\% CI, 7.6\%, 14.4\%), and likely lower than the prevalence reported by the 2010 World Bank Health Nutrition and Population Statistics for Syria, which reported $27.9 \%{ }^{7,15,16}$ The overall prevalence of wasting (5.57\% [ $95 \% \mathrm{CI}, 3.19 \%, 7.94 \%])$ in the 0 to 59 months age group was also medium severity by WHO thresholds. This is similar to the prevalence of wasting reported by the IOM's nutritional profile, which reported $4.1 \%$ (95\% CI, 3.6\%, 4.7\%), and higher than the 2013-2014 nutritional profile, which reported $0.8 \%(95 \% \mathrm{CI}, 0.3 \%, 2.2 \%)$ and likely lower than the 2010 World Bank's statistics, which reported $11.5 \%$. 7,15,16 
The prevalence of overweight ( $16.36 \%$ [95\% CI, 10.71\%, 22\%]) in the 0 to 59 months age group was high severity by WHO thresholds. This was lower than the prevalence reported by the 2010 World Bank's statistics, which found prevalence of overweight to be $17.9 \% .{ }^{16}$ Although WHO does not define prevalence thresholds for ages $>59$ months, we found the prevalence of stunting to be $7 \%(95 \% \mathrm{CI}, 3.57 \%, 10.85 \%)$, severe wasting $4 \%$ (95\% CI, 0.98\%, 6.23\%), wasting 5\% (95\% $\mathrm{Cl}, 2.04 \%, 8.26 \%)$, overweight $7 \%$ ( $95 \% \mathrm{CI}, 3.18 \%, 10.21 \%$ ), and underweight $5 \%$ ( $95 \% \mathrm{CI}, 1.26 \%, 7.88 \%$ ).

This data is thus interesting, as all of the prevalence values reported by the World Bank Health Nutrition and Population Statistics for Syria in 2010, which reflects preconflict data, appear worse than that reported by subsequent nutritional profiles.

Furthermore, the 2013-2014 nutritional profile suggested rates of acute malnutrition (defined by weight-forheight $z$ scores and prevalence of edema) in Syrian refugee children, who were between 6 to 59 months of age, were $<5 \%$ in all regions and not increased relatively or increased absolutely by the WHO's standards, although this survey did identify significantly higher levels of anemia. ${ }^{7}$

Those in the 0 to 59 months age group were also more likely to be stunted and overweight, which suggests greater impact on the younger population, either due to an intrinsically increased susceptibility, to greater length of exposure to their current environmental circumstance, or to both. These findings may be attributed to poorly balanced diets. However, it is possible that our measurements of weight were inflated by winterwear, as noted in the description of this study's limitations below. If this were the case, our study may potentially reflect grossly normal weights in this population, although we would not anticipate any significant impact on the reported prevalence of stunting.

As the Syrian conflict is still ongoing and refugee families remain displaced, poverty and food insecurity remain a high concern. In the time since these prior assessments were completed, the number of Syrian refugees in host communities continues to increase in Jordan, resulting in increased strain on public services and rising prices for rent and basic goods, with increased levels of vulnerability and food insecurity for refugees. ${ }^{17}$ There have also been policy changes regarding refugees in Jordan. Beginning in January 2018, the Jordanian government removed subsidies for several basic food items for refugees. ${ }^{18}$ Refugee households have had to significantly increase the resources they spend on food, and compared with only $23 \%$ in $2016,44 \%$ of households spend more than $40 \%$ of total expenditures on food, which may be in part a result of this removal of subsidies. ${ }^{18}$ According to the UNHCR, food security also decreased from 2016-2018 for refugees living in host communities in Jordan. Only $20 \%$ of registered Syrian households were food secure, compared with $28 \%$ in $2016 .{ }^{18}$ Furthermore, in February 2018, changes in government regulations prevented Syrians in Jordan from accessing the noninsured rate for health care and instead had to pay $80 \%$ of the "foreigner rate." This translated to a two-to5 -fold increase in health-associated costs. However, as of April 2019, the Jordanian government reversed this policy so that Syrians could access subsidized health care. ${ }^{17}$
There were several limitations to this study. First, this was a convenience sample, which does limit the strengths of inferences that can be made. It is possible that malnourished children were more likely than healthy children to present to the clinic. It is also possible that children may have been included more than once, as there were no measures in place to account for repeat visits by a child. This is because that data for this study were collected during a 6-day mission trip, where health care providers were volunteers, clinics were overwhelmed, and documentation was minimal, which did lead to other limitations as well. Due to the difficulties with data collection, standardization was not attainable, and the margin of error was thus increased. These include potential for imprecise reported age, user-dependent variation in anthropometric data collection, inaccuracies in true weight given weight-clothing, inability to include all children who presented to clinics, and inability to control for repeat data points.

\section{Conclusion}

This study shows that the Syrian refugee crisis may be having a significant effect on childhood nutrition and possibly resulting in wasting and stunting in Syrian refugee children living in Jordan. These findings should be concerning in the light of the known food insecurity and lack of food diversity that refugees face. We encourage further studies to be completed to provide a comprehensive assessment of the current nutrition status of Syrian children. As the crisis continues, it is of utmost importance that relief and resettlement efforts persist, with an emphasis on making sure Syrian children have access to adequate and nutritious food.

\section{Study Funding}

This research was supported by a scholarly award from Research, Innovation \& Scholarly Endeavors at USF Health, Morsani College of Medicine.

\section{Conflict of Interest}

A.V. is a member of the Syrian American Medical Society. N.K. is a member of the Syrian American Medical Society. A.K. has no relevant disclosures. L.M. has no relevant disclosures. M.A. is a member of the Syrian American Medical Society.

\section{Acknowledgments}

We thank the SAMS for their support and logistical assistance. We also thank Dr. Julia Fordham and Dr. Rania Haydar for their assistance with data collection, Yara ElMowafy for assistance in planning, and Dr. Luay Alkotob for his guidance.

\section{References}

1 World Vision Staff. Syrian refugee crisis: facts, FAQs, and how to help. Accessed September 1, 2019 at: https://www.worldvision. org/refugees-news-stories/syrian-refugee-crisis-facts

2 United Nations High Commissioner for Refugees. Syria Regional Refugee Response. Accessed September 1, 2019 at: https://data2. unhcr.org/en/situations/syria 
3 World Health Organization U.N.H.C.R. International Federation of Red Cross World Food Programme. The management of nutrition in major emergencies. Geneva: World Health Organization; 2000

4 USAID/CIDI. Organizations working in Syria and assisting Syrian refugees. 2017 Accessed Sep 01, 2019 at: https://www.usaid. gov/crisis/syria

5 Berti B. The Syrian refugee crisis: regional and human security implications. Strategic Assessment 2015;17(04):44-47

6 Norman KP. Access to legal residency for refugees in the Middle East: bureaucracy, deterrence, and prolonged impermanence. 2016 Accessed Sep 1, 2019 at: https://www.mei.edu/publications/access-legal-residency-refugees-middle-east-bureaucracydeterrence-and-prolonged

7 Hossain SM, Leidman E, Kingori J, Al Harun A, Bilukha OO. Nutritional situation among Syrian refugees hosted in Iraq, Jordan, and Lebanon: cross sectional surveys. Confl Health 2016; 10:26

8 FAO UNICEF WFP WHO. The State of Food Security and Nutrition in the World: Building Climate Resilience for Food Security and Nutrition. Rome: FOA; 2018

9 World Health Organization. WHO Anthro Survey Analyser Quick Guide. Geneva: World Health Organization; 2019

10 Borghi E, Blossner M, de Onis M, et al. WHO Anthro Survey Analyser: Software for Analysing Survey Anthropometric Data for Children Under 5 Years of Age. Built-in software edition. Version 1.0. Geneva: World Health Organization; 2018

11 World Health Organization. WHO AnthroPlus for Personal Computers Manual: Software for Assessing Growth of the World's Children and Adolescents. Geneva: WHO; 2009

12 World Health Organization. Physical Status: The Use and Interpretation of Anthropometry. Report of a WHO Expert Committee. Geneva: WHO; 1995

13 de Onis M, Borghi E, Arimond M, et al. Prevalence thresholds for wasting, overweight and stunting in children under 5 years. Public Health Nutr 2019;22(01):175-179

14 SPSS Statistics for Windows. Armonk, NY: IBM Corp; 2021

15 Pernitez-Agan S, Wickramage K, Yen C, Dawson-Hahn E, Mitchell T, Zenner D. Nutritional profile of Syrian refugee children before resettlement. Confl Health 2019;13:22

16 World Bank Group. Health nutrition and population statistics. Accessed Sep 1, 2019 at: https://databank.worldbank.org/reports. aspx? source=health-nutrition-and-population-statistics

17 United Nations High Commissioner for Refugees. 2019 Progress Report: 3RP Regional Refugee and Resilience Plan in Response to the Syria Crisis. UNHCR. 2019

18 World Food Programme and REACH. Jordan-Comprehensive Food Security and Assessment, 2018. Amman: World Food Programme; 2019 